\title{
Investigation of the neuroprotective effects of Lycium barbarum water extract in apoptotic cells and Alzheimer's disease mice
}

\author{
XINYU HU ${ }^{1}$, YIDI QU ${ }^{2}$, QIUBO CHU ${ }^{2}$, WENSHU $\mathrm{LI}^{2,3}$ and JIAN HE ${ }^{2,4}$ \\ ${ }^{1}$ Faculty of Clinical Medicine, Changchun Medical College, Changchun, Jilin 130031; ${ }^{2}$ School of Life Sciences, \\ Jilin University, Changchun, Jilin 130012; ${ }^{3}$ College of Art and Sciences, New York University Shanghai, Shanghai 200122; \\ ${ }^{4}$ Key Laboratory of Systems Biomedicine and Collaborative Innovation Center of Systems Biomedicine, \\ Shanghai Center for Systems Biomedicine, Shanghai Jiaotong University, Shanghai 200240, P.R. China
}

Received March 7, 2017; Accepted November 20, 2017

DOI: $10.3892 / \mathrm{mmr} .2017 .8310$

\begin{abstract}
Alzheimer's disease (AD) affects people worldwide and is caused by chronic and progressive damage to the central nervous system. Lycium barbarum (LB), a renowned functional food and medicinal plant in Southeast Asia, may possess protective effects against nerve injury. The present study aimed to investigate the neuroprotective effects of LB water extract in a differentiated (D)PC12 cellular apoptosis model induced by L-glutamic acid (L-Glu), and a mouse model of AD, induced by the combination of $\mathrm{AlCl}_{3}$ and D-galactose. $\mathrm{LB}$ markedly increased DPC12 cell survival against L-Glu induced damage by increasing cell viability, reducing the apoptosis rate and G1 phase arrest, suppressing intracellular reactive oxygen species accumulation, blocking $\mathrm{Ca}^{2+}$ overload and preventing mitochondrial membrane potential depolarization. LB additionally normalized the expression levels of apoptosis regulator Bcl-2, apoptosis regulator BAX, and cleaved caspase-3, -8 and -9 in L-Glu exposed cells. In the AD mouse model, LB increased the amount of horizontal and vertical movement in the autonomic activity test, improved endurance time in the rotarod test and decreased escape latency time in the Morris water maze test. Additionally, the levels of acetylcholine and choline acetyltransferase were significantly increased in the serum and hypothalamus in the LB-treated AD mice. These data suggested that LB may exert neuroprotective effects and may aid in preventing neurodegenerative disease.
\end{abstract}

Correspondence to: Dr Wenshu Li, College of Art and Sciences, New York University Shanghai, 1555 Century Avenue, Shanghai 200122, P.R. China

E-mail:wenshu.li@nyu.edu

Dr Jian He, Key Laboratory of Systems Biomedicine and Collaborative Innovation Center of Systems Biomedicine, Shanghai Center for Systems Biomedicine, Shanghai Jiaotong University, 800 Dongchuan Road, Shanghai 200240, P.R. China

E-mail: jih003@sjtu.edu.cn

Key words: Lycium barbarum, neuroprotection, apoptosis, Alzheimer's disease, mitochondria

\section{Introduction}

Alzheimer's disease (AD) is caused by chronic and progressive damage to the central nervous system. It has affected 60-65\% of people worldwide (1). One of the primary clinical manifestations reported in $\mathrm{AD}$ is a decline in cognitive function. Patients with AD frequently succumb to the development of a pulmonary embolism (2). However, the exact pathomechanism of $\mathrm{AD}$ remains to be completely elucidated. The induction of apoptosis in neurons has been proposed to be a potential theory behind AD pathogenesis (3). Neurons exhibit apoptotic features during the development of $\mathrm{AD}$, including apoptotic mitochondrial alterations (4). During mitochondrial apoptosis, the accumulation of intracellular reactive oxygen species (ROS) and calcium $\left(\mathrm{Ca}^{2+}\right)$ overload is observed (5), which is responsible for functional and structural damage to brain tissues. For research studies, injection of D-galactose (D-gal) in combination with intragastric treatment with $\mathrm{AlCl}_{3}$ successfully decreased memory ability and has been used to establish an animal model of AD (6).

Despite the considerable scientific manpower and resources being devoted to developing novel AD therapies, there are no adequate treatment options at present. Due to their various biological responses, natural products have become a novel repository for drug screening (7). For example, Sparassis crispa polysaccharides exert neuroprotective effects against L-glutamate (L-Glu)-induced cell damage via the mitochondrial pathway of apoptosis (8). Hericium erinaceus has been confirmed to have neuroprotective properties in L-Glu-induced apoptotic differentiated (D)PC12 cells and mouse models of AD (9).

Lycium barbarum (LB), a renowned functional food and medicinal plant from Southeast Asia, exhibits immunoregulatory and neuroprotective properties (10). It has been reported that polysaccharides separated from LB can prevent the apoptosis of 6-hydroxydopamine-induced PC12 cells, in part through regulation of the ROS-nitric oxide pathway (11). LB polysaccharides can also protect retinal ganglion cells against acute ocular hypertension induced ischemic injury (12). Furthermore, LB polysaccharides exhibit neuroprotective effects in differentiated PC12 cells against L-Glu induced toxicity through the regulation of the mitochondrial pathway 
of apoptosis (8). The present study aimed to further investigate the neuroprotective effects of LB and its underlying mechanisms in DPC12 cells exposed to L-Glu and in an AD mouse model established by $\mathrm{AlCl}_{3}$ and $\mathrm{D}$-gal. The results suggested that LB may possesses beneficial effects against L-Glu-induced toxicity via the mitochondrial pathway of apoptosis. The therapeutic effects of LB on AD were confirmed through the AD mouse model, and provide evidence for $\mathrm{LB}$ as a potential functional food that may be administered to patients with a neurodegenerative disease.

\section{Materials and methods}

LB water extract preparation. LB (acquired from Beijing Tongren Tang Co., Ltd., Beijing, China) extract was obtained by soaking in double distilled water at $90^{\circ} \mathrm{C}$ for $2 \mathrm{~h}$ twice. It was subsequently concentrated and freeze-dried for further experiments. LB was analyzed via 3,5-dinitrosalicylic acid colorimetric estimation (13), phenol-sulfuric acid determination (13) and the Kjeldahl method (14). The constituents were as follows: $9.2 \%$ total sugar; $1.9 \%$ reducing sugar; and $9.4 \%$ total protein.

Cell culture. PC12 cells (American Type Culture Collection, Manassas, VA, USA) were cultured in Dulbecco's modified Eagle's medium (DMEM; Invitrogen; Thermo Fisher Scientific, Inc., Waltham, MA, USA) and supplemented with $10 \%$ fetal bovine serum (FBS; Invitrogen; Thermo Fisher Scientific, Inc.), 5\% horse serum (Invitrogen; Thermo Fisher Scientific, Inc.), $1 \%$ penicillin and $1 \%$ streptomycin, in a humidified atmosphere containing $5 \% \mathrm{CO}_{2}$ at $37^{\circ} \mathrm{C}$. Nerve growth factor (NGF; Sigma-Aldrich; Merck KGaA, Darmstadt, Germany) at $20 \mathrm{ng} / \mathrm{ml}$ dissolved in DMEM (Invitrogen; Thermo Fisher Scientific, Inc.) with 1\% FBS (Invitrogen; Thermo Fisher Scientific, Inc.) was applied for $48 \mathrm{~h}$ to differentiate cells.

MTT assay. DPC12 cells were seeded in 96-well plates at $1 \times 10^{4}$ cells/well. No LB, 200 or $400 \mu \mathrm{g} / \mathrm{ml} \mathrm{LB}$ was applied to pre-incubate DPC12 cells for $3 \mathrm{~h}$ prior to $24 \mathrm{~h}$ exposure to $20 \mathrm{mM}$ of L-Glu. An MTT assay (Sigma-Aldrich; Merck $\mathrm{KGaA}$ ) was used to determine cell viability. Following incubation with MTT solution $(0.5 \mathrm{mg} / \mathrm{ml})$ for $3 \mathrm{~h}$ at $37^{\circ} \mathrm{C}$ in darkness, $100 \mu \mathrm{l}$ dimethyl sulfoxide was added to dissolve the purple formazan. A micro-plate reader (Bio-Rad Laboratories, Inc., Hercules, CA, USA) was used to detect the absorbance at $490 \mathrm{~nm}$. Viability values of treated cells were expressed as a percentage of that of corresponding control cells.

Assessment of caspase activity. DPC12 cells were seeded in a six-well plate at a density of $3 \times 10^{5}$ cells/well. DPC12 cells were treated with no LB, 200 or $400 \mu \mathrm{g} / \mathrm{ml} \mathrm{LB}$ for $3 \mathrm{~h}$, prior to co-incubation with or without $20 \mathrm{mM} \mathrm{L-Glu}$ for a further $24 \mathrm{~h}$. The activity of caspase- 3 in the cell lysate was measured with a caspase-3 activity assay kit (Nanjing Jiancheng Bioengineering Institute, Nanjing, China).

Assessment of apoptotic rate and the cell cycle. DPC12 cells were seeded in a six-well plate at a density of $3 \times 10^{5}$ cells/well. No LB, 200 or $400 \mu \mathrm{g} / \mathrm{ml}$ LB was applied to DPC12 cells for $3 \mathrm{~h}$, prior to $24 \mathrm{~h}$ exposure to $20 \mathrm{mM} \mathrm{L}-\mathrm{Glu}$. The apoptotic rate and alterations in the cell cycle were measured using a flow cytometer (FC500; Beckman Coulter, Inc., Brea, CA, USA) and analyzed using FlowJo 7.6 software (Tree Star, Inc., Ashland, OR, USA). For the determination of apoptotic rate, treated cells were stained with propidium iodide (PI) and Annexin V (Annexin V-fluorescein isothiocyanate/PI double staining apoptosis detection kit; G003; Nanjing Jiancheng Bioengineering Institute) at room temperature in darkness for $20 \mathrm{~min}$. In a separate experiment, alterations in the cell cycle were determined in treated cells stained with PI at room temperature in darkness for $10 \mathrm{~min}$.

Mitochondrial membrane potential (MMP) analysis. DPC12 cells were seeded in a six-well plate at a density of $2 \times 10^{5}$ cells/well. DPC12 cells were pretreated with no LB, 200 or $400 \mu \mathrm{g} / \mathrm{ml} \mathrm{LB}$ for $3 \mathrm{~h}$, and subsequently exposed to $20 \mathrm{mM}$ L-Glu for $12 \mathrm{~h}$. Following staining with $2 \mu \mathrm{g} / \mathrm{ml} \mathrm{JC}-1$ (Sigma-Aldrich; Merck KGaA) in darkness for $15 \mathrm{~min}$, alterations in green (excitation, $490 \mathrm{~nm}$ and emission, $530 \mathrm{~nm}$ ) and red, (excitation, $540 \mathrm{~nm}$ and emission, $590 \mathrm{~nm}$ ) fluorescence were detected using a fluorescent microscope (magnification, x20; TE2000; Nikon Corporation, Tokyo, Japan).

Intracellular $\mathrm{Ca}^{2+}$ and $\mathrm{ROS}$ concentration analysis. DPC12 cells were seeded in a six-well plate $\left(2 \times 10^{5}\right.$ cells/well $)$ and were pretreated with no LB, 200 or $400 \mu \mathrm{g} / \mathrm{ml}$ of LB for $3 \mathrm{~h}$, prior to co-incubation with or without $20 \mathrm{mM} \mathrm{L-Glu}$ for $12 \mathrm{~h}$. The treated cells were stained with $5 \mu \mathrm{M}$ Fluo-4-AM (Molecular Probes; Thermo Fisher Scientific, Inc.) to detect $\mathrm{Ca}^{2+}$ levels, or $10 \mu \mathrm{M}$ of 2,7'-dichlorofluorescein diacetate (Sigma-Aldrich; Merck $\mathrm{KGaA}$ ) to detect ROS levels. After 15-min staining in darkness at $37^{\circ} \mathrm{C}$, cells were washed with HBSS for three times. Alterations in green fluorescence intensity were subsequently analyzed by flow cytometry (FC500; Beckman Coulter, Inc.). FlowJo v7.6 software (Tree Star, Inc.) was used to analyze fluorescence intensity.

Western blot analysis. DPC12 cells were seeded in a six-well plate at a density of $3 \times 10^{5}$ cells/well. Cells were pretreated with 200 or $400 \mu \mathrm{g} / \mathrm{ml} \mathrm{LB}$ for $3 \mathrm{~h}$, and subsequently co-incubated with or without $20 \mathrm{mM} \mathrm{L-Glu}$ for a further $24 \mathrm{~h}$. Treated cells were lysed with radioimmunoprecipitation assay buffer (Sigma-Aldrich; Merck KGaA) containing 1\% protease inhibitor cocktail (Sigma-Aldrich; Merck KGaA). A Bicinchoninic Acid protein assay kit (Merck KGaA) was used to determine the concentration of the lysed cell protein according to the manufacturer's protocols. Proteins $(40 \mu \mathrm{g})$ were separated on a 10-12\% SDS-PAGE gel. Following electrophoretic transfer, the nitrocellulose membranes (0.45 $\mu \mathrm{m}$; Bio Basic, Inc., Markham, ON, Canada) were exposed to primary antibodies including cleaved caspase-3 (9662), caspase-8 (9746) and caspase-9 (9502), apoptosis regulator Bcl-2 (Bcl-2; 2872), apoptosis regulator BAX (Bax; 2772) and GAPDH (5174; all obtained from Cell Signaling Technology, Inc., Danvers, MA, USA) at a dilution of 1:2,000 for $12 \mathrm{~h}$ at $4^{\circ} \mathrm{C}$. The membranes were subsequently incubated with horseradish peroxidase-conjugated goat anti-rabbit secondary antibodies (sc-3836; Santa Cruz Biotechnology, Inc., Dallas, TX, USA) accordingly at a dilution of 1:500, for $2 \mathrm{~h}$ at room temperature. An enhanced chemiluminescence 


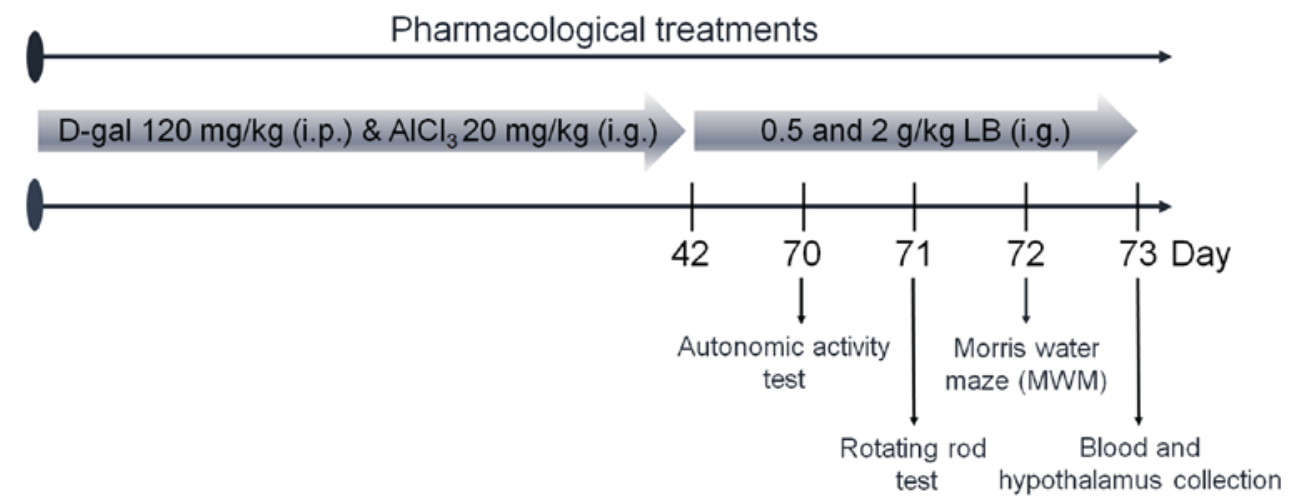

Figure 1. Experimental protocol for the establishment of the Alzheimer's disease mouse model and drug administration. D-Gal, D-galactose; LB, Lycium barbarium; i.g., intragastric; i.p., intraperitoneal.

detection kit (Merck KGaA) was used to detect the chemiluminescence of blots and ImageJ v1.38 software (National Institutes of Health, Bethesda, MD, USA) was used to quantify the intensity.

Animal care and drug treatment process. The experimental protocol was approved by the Institutional Animal Ethics Committee of Jilin University (Changchun, China). Male BALB/c mice (20-22 g; 8 weeks old; $n=40$ ) were housed in groups of 6 in transparent cages and maintained on a 12-h light/dark cycle at $23 \pm 1^{\circ} \mathrm{C}$ with water and food available ad libitum. Mice were treated intragastrically with $20 \mathrm{mg} / \mathrm{kg} \mathrm{AlCl}$ and subcutaneously injected with $120 \mathrm{mg} / \mathrm{kg}$ D-gal once daily for 6 weeks to establish the AD model, which was determined via a Morris water maze test as described below. AD mice were treated orally with normal saline, 0.5 or $2.0 \mathrm{~g} / \mathrm{kg}$ LB for 4 weeks $(\mathrm{n}=10)$. Non-induced BALB/c mice $(n=10)$ were treated with normal saline as a control. Following the final treatment, mice underwent behavioral testing. The protocol is presented in Fig. 1.

\section{Behavioral tests}

Autonomic activity test. As described previously (9), the horizontal and vertical locomotor activities of the mice were recorded for $5 \mathrm{~min}$ following placement into squares.

Fatigue rotarod test. Following a previous study protocol (9), training was repeated three times and mice were placed on the turning device (Chengdu Techman Software Co., Ltd., Chengdu, China) at a speed of $20 \mathrm{rpm}$ and their time until exhaustion was recorded.

Morris water maze. Following a training period of 5 days, mice were placed in an open swimming arena with a depth of $10 \mathrm{~cm}$ and a temperature of $25 \pm 2{ }^{\circ} \mathrm{C}$. Following a previous study protocol (9), the time spent within the target quadrant over a $120 \mathrm{sec}$ probe test period was recorded.

Measurement of acetylcholine (ACh) and choline acetyltransferase (ChAT) levels. Following behavioral testing, blood from caudal veins of mice and the hypothalamus were collected. The hypothalamus was homogenized in saline (1-5 w/v). ELISA kits were used according to manufacturer's protocols to analyze the levels of Ach and ChAT (A105-1 and A079-1, respectively, Nanjing Jiancheng Bioengineering Institute) in the serum and hypothalamus.

Statistical analysis. The data are expressed as the mean \pm standard deviation. A one-way analysis of variance was used to detect statistical significance followed by post hoc Dunn's test. $\mathrm{P}<0.05$ was considered to indicate a statistically significant difference.

\section{Results}

$L B$ improves cell viability, inhibited apoptotic rate and normalized cell cycle. LB alone failed to influence the levels of cell proliferation (Fig. 2A). In L-Glu treated cells, 3 h pretreatment with 200 and $400 \mu \mathrm{g} / \mathrm{ml}$ LB improved cell viability by $>20 \%$ ( $\mathrm{P}<0.05$; Fig. 2A). Treatment with LB suppressed caspase- 3 activity by $>30 \%$ in L-Glu treated cells $(\mathrm{P}<0.05$; Fig. 2B). L-Glu caused a cellular apoptotic rate of $17.2 \%$ of in DPC12 cells, whereas LB reduced the rate of apoptosis by $\sim 50 \%$ (Fig. 2C). Exposure to LB for $24 \mathrm{~h}$ markedly reversed G1 arrest in DPC12 cells induced by L-Glu (Fig. 2D).

$L B$ reverses mitochondrial apoptosis. Intense green fluorescence was observed in 12-h L-Glu-incubated cells, indicating MMP depolarization. Comparatively, LB pre-incubation strongly enhanced the ratio of red to green fluorescence, suggesting a beneficial effect on mitochondrial function $(\mathrm{P}<0.05$; Fig. 3A). Furthermore, by comparison with L-Glu incubated cells, LB markedly suppressed the intracellular levels of ROS (Fig. 3B) and $\mathrm{Ca}^{2+}$ (Fig. 3C) following $3 \mathrm{~h}$ pretreatment in combination with $12 \mathrm{~h}$ co-incubation with L-Glu.

$L B$ reverses alterations in pro- and anti-apoptotic protein expression. A significant decrease in $\mathrm{Bcl}-2$ expression levels, and a significant increase in the expression levels of Bax, and cleaved caspase-3, -8 and -9 was observed in L-Glu-treated DPC12 cells $(\mathrm{P}<0.05$; Fig. 4). DPC12 cells pre-treated with LB at doses of 200 and $400 \mu \mathrm{g} / \mathrm{ml}$ exhibited a significant increase in the levels of Bcl-2 expression, and the expression levels of Bax, and cleaved caspase-3, -8 and -9 were significantly decreased compared with cells treated with L-Glu only ( $\mathrm{P}<0.05$; Fig. 4). 

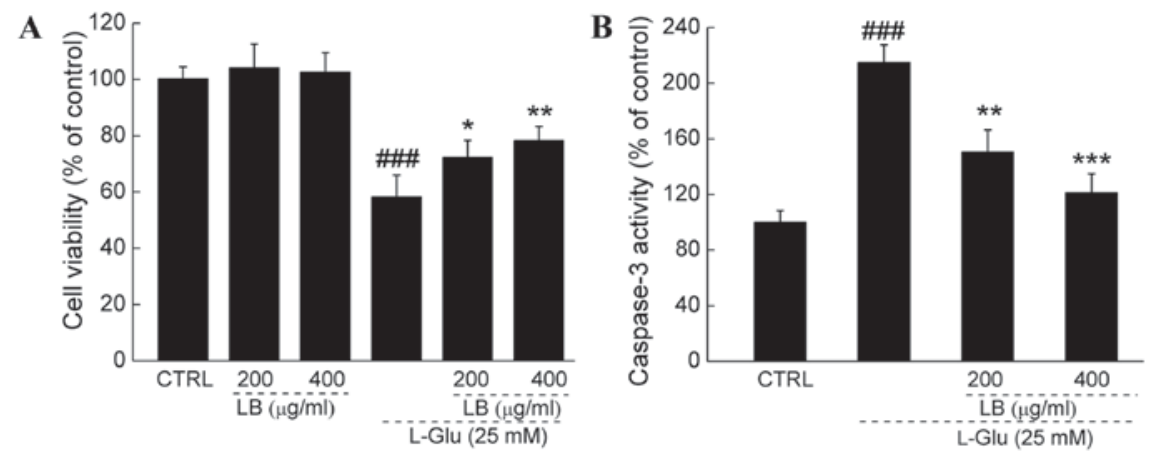

C

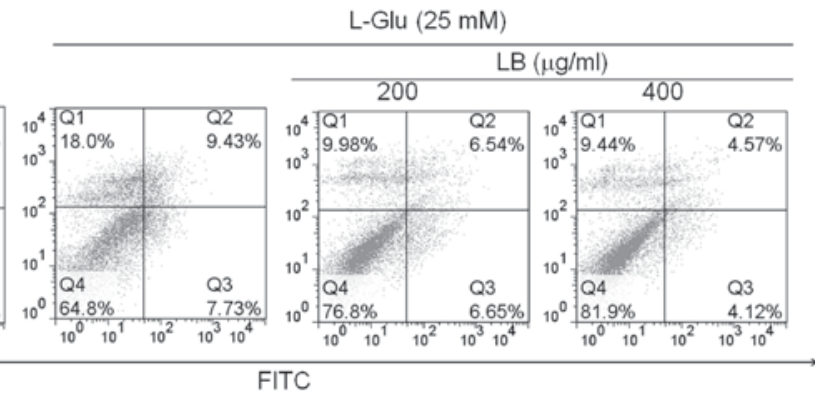

D

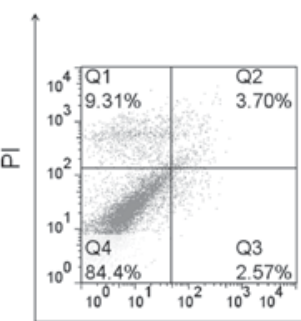

L-Glu $(25 \mathrm{mM})$

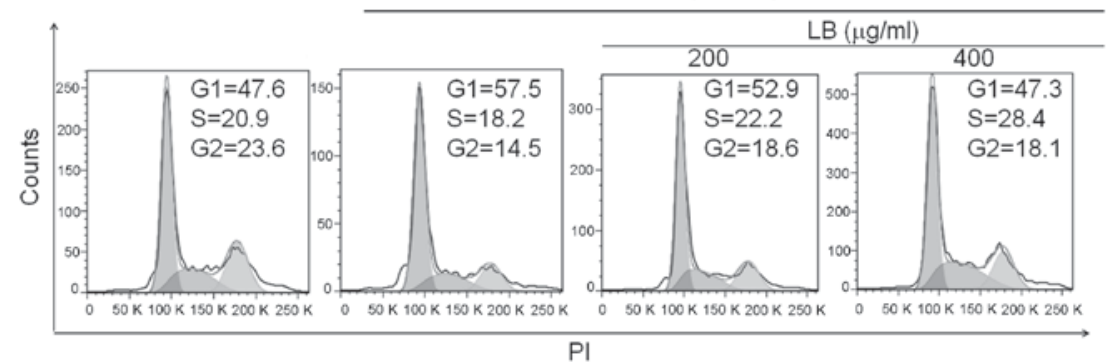

Figure 2. Effects of LB on cell viability and apoptosis. (A) LB increased cell viability in DPC12 cells exposed to L-Glu for $24 \mathrm{~h}$. (B) LB reduced the levels of caspase-3 activity in DPC12 cells exposed to L-Glu for $24 \mathrm{~h}$. Data are expressed as the mean \pm standard deviation ( $\mathrm{n}=10)$. ${ }^{\# \# \#} \mathrm{P}<0.001 \mathrm{vs}$. CTRL; ${ }^{*} \mathrm{P}<0.05$, ${ }^{* *} \mathrm{P}<0.01,{ }^{* * *} \mathrm{P}<0.001$ vs. L-Glu only. (C) LB decreased the L-Glu-induced increase in apoptotic rate in DPC12 cells ( $\mathrm{n}=6$ ). (D) LB reduced the accumulation of cells in the G1 phase (n=6). LB, Lycium barbarum; DPC12, differentiated PC12; L-Glu, L-glutamic acid; CTRL, non-induced control; PI, propidium iodide; FITC, fluorescein isothiocyanate.

Effects of $L B$ on $A D$ mouse behavior. Following 4 weeks of treatment with $\mathrm{LB}$, the quantity of horizontal and vertical movements was increased by $>25 \%(\mathrm{P}<0.01$; Fig. 5A and $\mathrm{B})$ by comparison with $\mathrm{AD}$ mice. Endurance time was increased in the rotarod test by $30 \%$ following 4 weeks of treatment with $\mathrm{LB}$, by comparison with $\mathrm{AD}$ mice $(\mathrm{P}<0.01$; Fig. $5 \mathrm{C})$. The Morris water maze test is commonly applied to evaluate the effects of a drug on the learning and memory of an animal. Escape latency time increased by over two-fold in AD mice compared with the wild-type control group ( $\mathrm{P}<0.001$; Fig. 5D). Treatment with $\mathrm{LB}(0.5$ and $2 \mathrm{~g} / \mathrm{kg})$ returned the escape latency time into the normal range $(\mathrm{P}<0.05$; Fig. 5D).

$L B$ upregulates ACh and ChAT levels in the serum and hypothalamus. Significantly reduced serum and hypothalamic levels of ACh and ChAT were observed in $\mathrm{AD}$ mice compared with the control group $(\mathrm{P}<0.001$; Fig. 6), suggesting that central cholinergic function was disturbed by $\mathrm{AlCl}_{3}$ and $\mathrm{D}$-gal. Comparatively, the levels of ACh and ChAT in the serum and hypothalamus were significantly increased $(\mathrm{P}<0.05$; Fig. 6) following 4 weeks of treatment with $\mathrm{LB}$, demonstrating the ability of LB to improve central cholinergic system function in $\mathrm{AD}$ mice.

\section{Discussion}

LB is different from other potential therapies currently being investigated for $\mathrm{AD}$. The water extract of this functional food contains natural active ingredients that have been consumed safely in Southeast Asia for centuries. The present study confirmed the neuroprotective effects of LB in in vitro and in vivo models. $\mathrm{LB}$ was demonstrated to increase cell viability, inhibit cellular apoptosis, ameliorate mitochondrial apoptotic alterations and normalize behaviors in $\mathrm{AD}$ mice.

$\mathrm{PC} 12$ cells are able to differentiate into neuron-like cells that form clear synapses and produce nerve-associated proteins (15). Glutamate is reported to be an excitatory neurotransmitter in the central nervous system; however, excessive levels are responsible for excitoxicity (16). Glutamate receptors are excessively activated in patients with neuropathological conditions, which induces neuronal death processes (17). In the present in vitro study, $25 \mathrm{mM}$ L-Glu was used to establish an apoptotic 

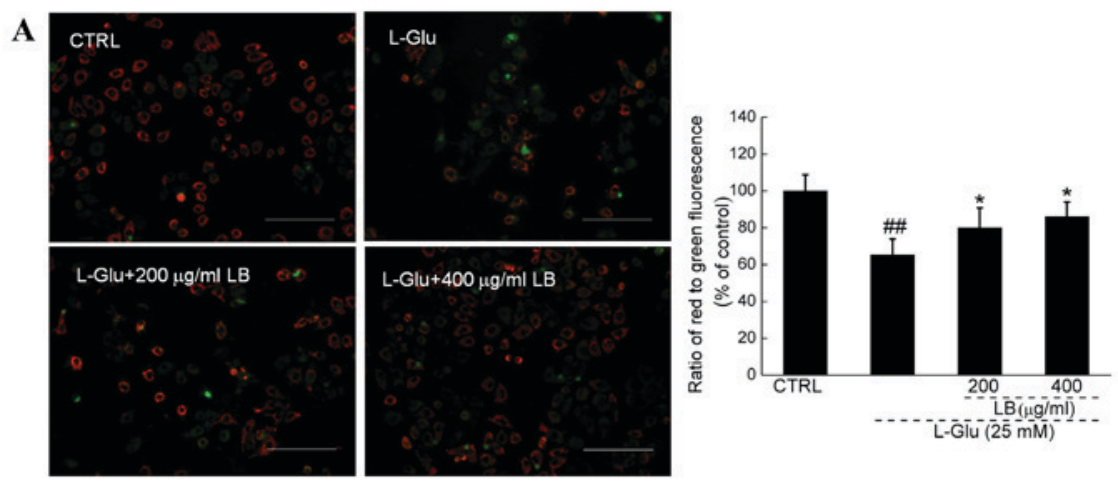

B

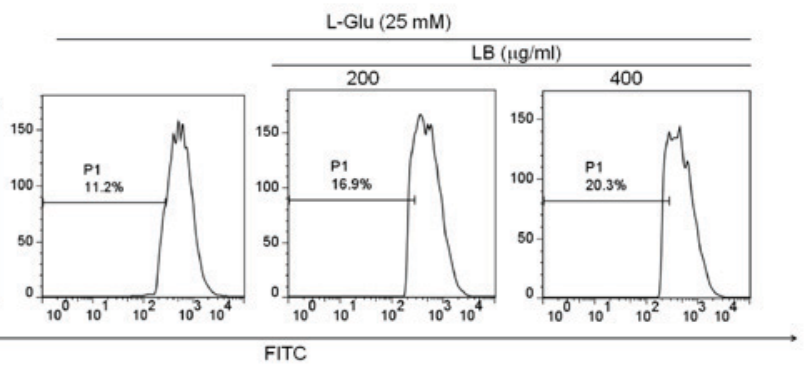

C

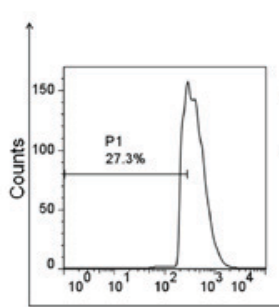

L-Glu (25 mM)

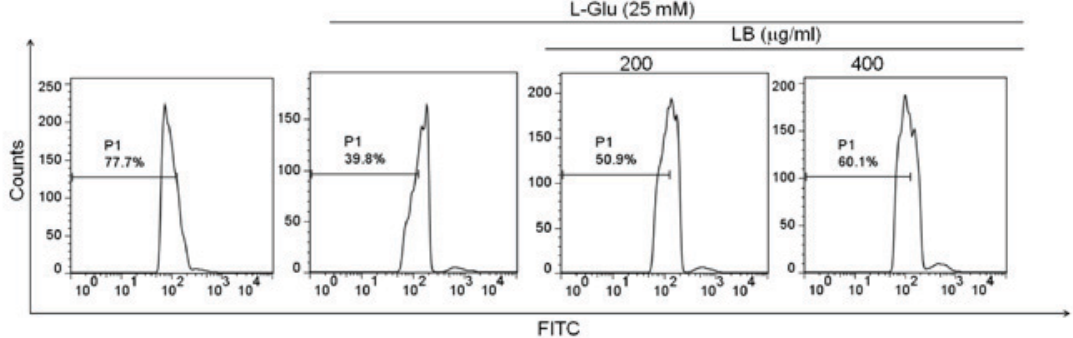

Figure 3. LB reverses mitochondrial apoptosis. (A) Pretreatment for $3 \mathrm{~h}$ with LB restored the MMP disruption induced by $12 \mathrm{~h} \mathrm{L-Glu}$ exposure. Scale bar, $100 \mu \mathrm{m}$. Data are expressed as the mean \pm standard deviation $(\mathrm{n}=6)$. ${ }^{\# \#} \mathrm{P}<0.01$ vs. CTRL; ${ }^{*} \mathrm{P}<0.05 \mathrm{vs}$. L-Glu only. (B) Pretreatment with LB (3 h) reduced the levels of intracellular reactive oxygen species in DPC12 cells exposed to L-Glu (n=6). (C) Pretreatment with LB ( 3 h) inhibited intracellular Ca ${ }^{2+}$ overload $(\mathrm{n}=6)$ in DPC12 cells exposed to L-Glu. LB, Lycium barbarum; MMP, mitochondrial membrane potential; L-Glu, L-glutamic acid; CTRL, non-induced control; DPC12, differentiated PC12; PI, propidium iodide.
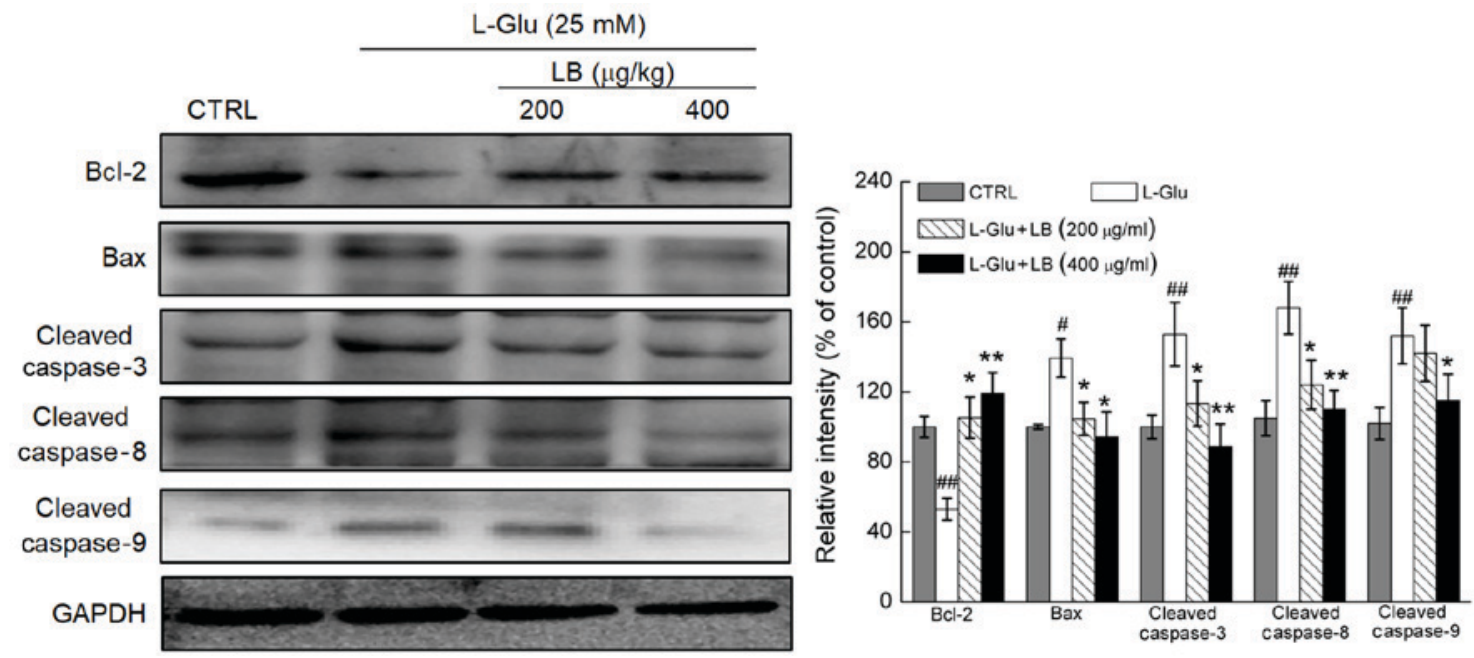

Figure 4. Pretreatment with LB $(3 \mathrm{~h})$ followed by $24 \mathrm{~h} \mathrm{~L}$-Glu co-exposure significantly increased the expression levels of Bcl-2 and reduced the expression levels of Bax, and cleaved caspase-3, -8 and -9 , compared with L-Glu-exposed cells. Quantification data of protein expression were normalized to GAPDH and expressed as the mean \pm standard deviation $(\mathrm{n}=6) .{ }^{*} \mathrm{P}<0.05$ and ${ }^{\# \#} \mathrm{P}<0.01$ vs. CTRL; ${ }^{*} \mathrm{P}<0.05$ and ${ }^{* *} \mathrm{P}<0.01$ vs. L-Glu only. LB, Lycium barbarum; L-Glu, L-glutamic acid; Bcl-2, apoptosis regulator Bcl-2; Bax, apoptosis regulator BAX; CTRL, non-induced control.

DPC12 cell model, in order to investigate the neuroprotective effects of LB. The data revealed that LB significantly suppressed L-Glu-induced $\mathrm{Ca}^{2+}$ overload and ROS accumulation. Energy metabolizing mitochondria are recognized to be 

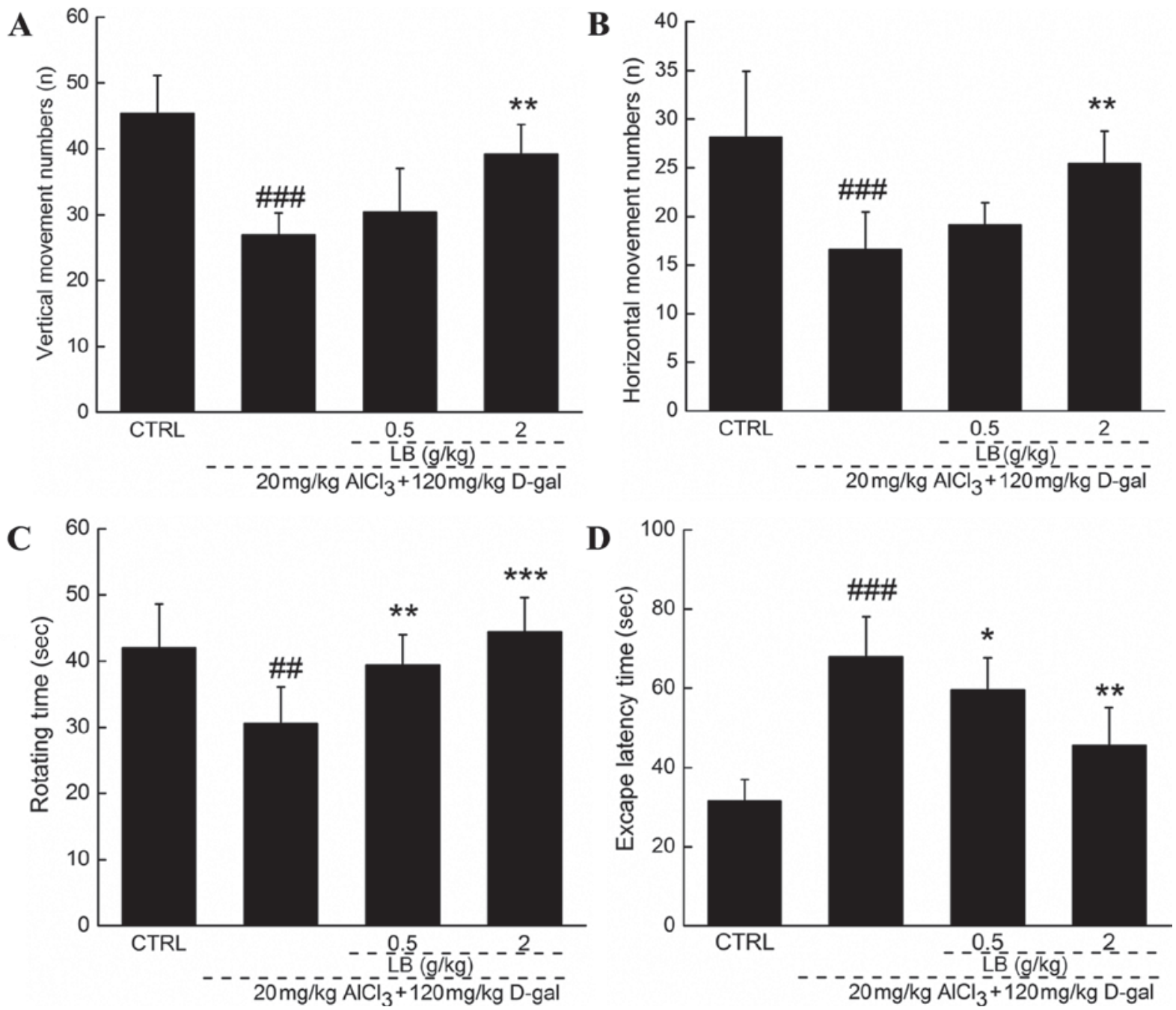

Figure 5. Effects of LB on behavior in the AD mouse model. Treatment with LB (4 weeks) significantly increased (A) horizontal movements and (B) vertical movements in the locomotor activity test, and (C) endurance time in the rotarod test, and (D) reduced escape latency time in the Morris water maze compared with AD mice. Data is expressed as a mean \pm standard deviation $(\mathrm{n}=10) .{ }^{\# \#} \mathrm{P}<0.05$ and ${ }^{\# \#} \mathrm{P}<0.001$ vs. CTRL; ${ }^{*} \mathrm{P}<0.05,{ }^{* * *} \mathrm{P}<0.01$ and ${ }^{* * *} \mathrm{P}<0.001$ vs. AD mice. LB Lycium barbarum; AD, Alzheimer's disease; CTRL, non-induced control; D-gal, D-galactose.
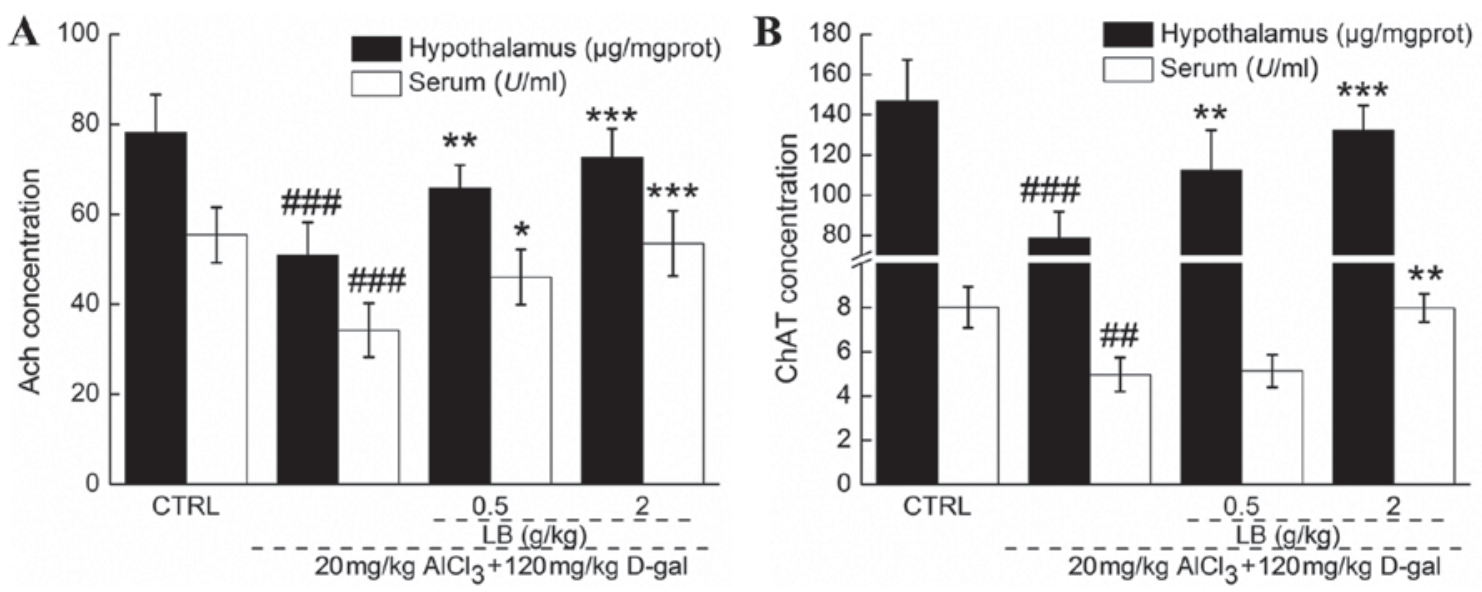

Figure 6. Effects of LB on serum and hypothalamic ACh and ChAT levels. Following 4 weeks of treatment with LB, levels of (A) ACh and (B) ChAT were significantly increased in the serum and hypothalamus. Data are expressed as the mean \pm standard deviation $(\mathrm{n}=10) .{ }^{\# \#} \mathrm{P}<0.01$ and ${ }^{\# \# \#} \mathrm{P}<0.001 \mathrm{vs}$. CTRL, $\mathrm{P}<0.05$, ${ }^{* *} \mathrm{P}<0.01$ and ${ }^{* * *} \mathrm{P}<0.001$ vs. Alzheimer's disease mice. ACh, acetylcholine; ChAT, choline acetyltransferase; LB, Lycium barbarum; D-gal, D-galactose.

$\mathrm{Ca}^{2+}$ hubs (18), and $\mathrm{Ca}^{2+}$ overload is responsible for mitochondrial depolarization, which in turn leads to further release of free radicals, particularly ROS (19). Intracellular free radicals are an essential factor during apoptosis, and thus they have 
become a target for the prevention of apoptosis (20). High ROS levels stimulate the opening of the mitochondrial permeability transition pore, which contributes to the activation of the mitochondrial pathway of apoptosis (21). Notably, a feedback loop between intracellular ROS levels and mitochondrial function has been demonstrated, with ROS accumulation inducing MMP dissipation, which further contributes to excessive ROS production (22). The results of the present study demonstrated that $\mathrm{LB}$ enhanced $\mathrm{Bcl}-2$ expression levels and reduced the expression levels of Bax, and cleaved caspase-3, -8 and -9 in L-Glu-exposed DPC12 cells. Bcl-2 family members, located in the outer mitochondrial membrane, serve as measures of mitochondrial function (23). The activation of caspase family members has a central role in neurodegeneration, particularly caspase-3 (24). MMP disruption activates the enzymatic apoptotic machinery of caspases, which are responsible for cellular fragmentation into apoptotic bodies (25). In response to extracellular stimuli, caspase- 8 directly activates caspase- 3 through the mitochondrial apoptotic pathway $(26,27)$. Mitochondria subsequently release cytochrome c into the cytoplasm, which is associated with the activation of caspase-9 (28). Caspase-3 is subsequently activated, which has a critical role in the mitochondrial apoptotic cascade, in part through the amplification of initiator caspase- 8 and -9 signals (29). Results indicate that LB-mediated neuroprotection against L-Glu induced DPC12 cell apoptosis may be associated with mitochondrial apoptotic signaling.

Due to the complexity of AD pathology, establishing a representative animal model for basic research is difficult (30). D-gal induces the swelling and dysfunction of brain cells (31), and aluminum promotes amyloid $\beta$ production in astrocytes (32), the two of which result in cognitive and memory dysfunction in animals (32). The combination of $\mathrm{AlCl}_{3}$ and $\mathrm{D}$-gal establishes a mouse model displaying AD-like behaviors which are more stabilized than that of $\mathrm{AlCl}_{3}$ or D-gal alone (6). $\mathrm{HB}$ has been confirmed to improve the cognition of mice in an $\mathrm{AlCl}_{3}$ and D-gal-induced AD model (9). Similarly, LB significantly alleviated the loss of memory and learning ability in AD mice. Furthermore, LB significantly increased the serum and hypothalamic expression levels of ACh and ChAT. Low levels of ChAT and ACh are consistently observed in brain tissues of patients with $\mathrm{AD}$, which is thought to be responsible for the decline in learning and memory abilities (33). H. erinaceus has been demonstrated to improve learning and memory abilities in AD mice via modulation of ACh and ChAT expression levels (9). Additionally, Flammulina velutipes polysaccharides increase the expression levels of ACh and ChAT in scopolamine-induced neuron damaged rats (34). The modulating effect of $\mathrm{LB}$ on $\mathrm{ACh}$ and $\mathrm{ChAT}$ expression levels suggests that its neuroprotective effects in AD mice may be mediated in part through the improvement of cholinergic function.

In conclusion, the neuroprotective effects of $\mathrm{LB}$ were successfully verified through a L-Glu-induced DPC12 apoptosis cell model and an $\mathrm{AlCl}_{3}$ and D-gal-induced $\mathrm{AD}$ mouse model. The present study revealed that this effect may be associated with modulation of the mitochondrial pathway of apoptosis and the cholinergic system. Thus, LB may be a potential candidate for the treatment or prevention of neurodegenerative disease.

\section{References}

1. Chang CH, Chen Y, Yew XX, Chen HX, Kim JX, Chang CC, Peng CC and Peng RY: Improvement of erinacine A productivity in Hericium erinaceus mycelia and its neuroprotective bioactivity against the glutamate-insulted apoptosis. LWT Food Sci Technol 65: 1100-1108, 2016.

2. Bermejo-Pareja F, Llamas Velasco S and Villarejo-Galende A: Alzheimer's disease prevention: A way forward. Revista Clínica Española (English Edition) 216: 495-503, 2016.

3. Rosello A, Warnes G and Meier UC: Cell death pathways and autophagy in the central nervous system and its involvement in neurodegeneration, immunity and central nervous system infection: To die or not to die-that is the question. Clin Exp Immunol 168: 52-57, 2012.

4. Karbowski $\mathrm{M}$ and Neutzner A: Neurodegeneration as a consequence of failed mitochondrial maintenance. Acta Neuropathol 123: 157-171, 2012.

5. Murphy E and Steenbergen C: Mechanisms underlying acute protection from cardiac ischemia-reperfusion injury. Physiol Rev 88: 581-609, 2008.

6. Luo Y, Niu F, Sun Z, Cao W, Zhang X, Guan D, Lv Z, Zhang B and $\mathrm{Xu} \mathrm{Y}$ : Altered expression of A beta metabolism-associated molecules from D-galactose/AlCl(3) induced mouse brain. Mech Ageing Dev 130: 248-252, 2009.

7. Li SP, Yang FQ and Tsim KW: Quality control of Cordyceps sinensis, a valued traditional Chinese medicine. J Pharm Biomed Anal 41: 1571-1584, 2006.

8. Hu S, Wang D, Zhang J, Du M, Cheng Y, Liu Y, Zhang N, Wang D and Wu Y: Mitochondria related pathway is essential for polysaccharides purified from sparassis crispa mediated neuro-protection against glutamate-induced toxicity in differentiated PC12 cells. Int J Mol Sci 17: pii: E133, 2016.

9. Zhang J, An S, Hu W, Teng M, Wang X, Qu Y, Liu Y, Yuan Y and Wang D: The Neuroprotective properties of Hericium erinaceus in glutamate-damaged differentiated PC12 cells and an Alzheimer's disease mouse model. Int J Mol Sci 17: pii: E1810, 2016.

10. Zareisedehizadeh S, Tan $\mathrm{CH}$ and Koh HL: A review of botanical characteristics, traditional usage, chemical components, pharmacological activities and safety of pereskia bleo (Kunth) DC. Evid Based Complement Alternat Med 2014: 326107, 2014

11. Gao K, Liu M, Cao J, Yao M, Lu Y, Li J, Zhu X, Yang Z and Wen A: Protective effects of Lycium barbarum polysaccharide on 6-OHDA-induced apoptosis in $\mathrm{PC} 12$ cells through the ROS-NO pathway. Molecules 20: 293-308, 2015.

12. Mi XS, Feng Q, Lo AC, Chang RC, Lin B, Chung SK and So KF: Protection of retinal ganglion cells and retinal vasculature by Lycium barbarum polysaccharides in a mouse model of acute ocular hypertension. PLoS One 7: e45469, 2012.

13. Zhang N, Li Q, Wang J and Teng L: Screening of Irpex lacteus mutant strains and optimizing fermentation conditions. J Food Agric Environ 12: 1213-1219, 2014.

14. Wang H, Pampati N, McCormick WM and Bhattacharyya L: Protein nitrogen determination by kjeldahl digestion and ion chromatography. J Pharm Sci 105: 1851-1857, 2016.

15. Su WT and Shih YA: Nanofiber containing carbon nanotubes enhanced PC12 cell proliferation and neuritogenesis by electrical stimulation. Biomed Mater Eng 26 (Suppl 1): S189-S195, 2015.

16. Shimmyo Y, Kihara T, Akaike A, Niidome T and Sugimoto H: Three distinct neuroprotective functions of myricetin against glutamate-induced neuronal cell death: Involvement of direct inhibition of caspase-3. J Neurosci Res 86: 1836-1845, 2008.

17. Cheriyan J, Balsara RD, Hansen $\mathrm{KB}$ and Castellino FJ: Pharmacology of triheteromeric N-Methyl-d-Aspartate receptors. Neurosci Lett 617: 240-246, 2016.

18. Feissner RF, Skalska J, Gaum WE and Sheu SS: Crosstalk signaling between mitochondrial $\mathrm{Ca} 2+$ and ROS. Front Biosci (Landmark Ed) 14: 1197-1218, 2009.

19. Bernardi P and Rasola A: Calcium and cell death: The mitochondrial connection. Subcell Biochem 45: 481-506, 2007.

20. Thatte U, Bagadey S and Dahanukar S: Modulation of programmed cell death by medicinal plants. Cell Mol Biol (Noisy-le-grand) 46: 199-214, 2000.

21. Christophe M and Nicolas S: Mitochondria: A target for neuroprotective interventions in cerebral ischemia-reperfusion. Curr Pharm Des 12: 739-757, 2006. 
22. Tang XQ, Feng JQ, Chen J, Chen PX, Zhi JL, Cui Y, Guo RX and Yu HM: Protection of oxidative preconditioning against apoptosis induced by $\mathrm{H} 2 \mathrm{O} 2$ in $\mathrm{PC} 12$ cells: Mechanisms via MMP ROS and Bcl-2. Brain Res 1057: 57-64, 2005.

23. Raisova M, Hossini AM, Eberle J, Riebeling C, Wieder T, Sturm I, Daniel PT, Orfanos CE and Geilen CC: The Bax/Bcl-2 ratio determines the susceptibility of human melanoma cells to CD95/Fas-mediated apoptosis. J Invest Dermatol 117: 333-340, 2001.

24. Luo M, Lu Z, Sun H, Yuan K, Zhang Q, Meng S, Wang F, Guo H, $\mathrm{Ju} \mathrm{X}$, Liu Y, et al: Nuclear entry of active caspase-3 is facilitated by its p3-recognition-based specific cleavage activity. Cell Res 20: 211-222, 2010.

25. Hippe D, Gais A, Gross U and Luder CG: Modulation of caspase activation by Toxoplasma gondii. Methods Mol Biol 470: 275-288, 2009

26. Lee SY, Cherla RP, Caliskan I and Tesh VL: Shiga toxin 1 induces apoptosis in the human myelogenous leukemia cell line THP-1 by a caspase-8-dependent, tumor necrosis factor receptor-independent mechanism. Infect Immun 73: 5115-5126, 2005.

27. Yang D, Yaguchi T, NakanoT and Nishizaki T: Adenosine-induced caspase-3 activation by tuning Bcl-XL/DIABLO/IAP expression in HuH-7 human hepatoma cells. Cell Biol Toxicol 26: 319-330, 2010.

28. Boucher D, Blais V, Drag M and Denault JB: Molecular determinants involved in activation of caspase 7. Biosci Rep 31: 283-294, 2011.
29. Espín R, Roca FJ, Candel S, Sepulcre MP, González-Rosa JM, Alcaraz-Pérez F, Meseguer J, Cayuela ML, Mercader N and Mulero V: TNF receptors regulate vascular homeostasis in zebrafish through a caspase-8, caspase-2 and P53 apoptotic program that bypasses caspase-3. Dis Model Mech 6: 383-396, 2013.

30. Hall AM and Roberson ED: Mouse models of Alzheimer's disease. Brain Res Bull 88: 3-12, 2012.

31. Salminen A, Haapasalo A, Kauppinen A, Kaarniranta K, Soininen $\mathrm{H}$ and Hiltunen $\mathrm{M}$ : Impaired mitochondrial energy metabolism in Alzheimer's disease: Impact on pathogenesis via disturbed epigenetic regulation of chromatin landscape. Prog Neurobiol 131: 1-20, 2015

32. Wang Z, Wei X, Yang J, Suo J, Chen J, Liu X and Zhao X: Chronic exposure to aluminum and risk of Alzheimer's disease: A meta-analysis. Neurosci Lett 610: 200-2 06, 2016.

33. Farkas E and Luiten PGM: Cerebral microvascular pathology in aging and Alzheimer's disease. Prog Neurobiol 64: 575-611, 2001.

34. Yang W, Yu J, Zhao L, Ma N, Fang Y, Pei F, Mariga AM and $\mathrm{Hu}$ Q: Polysaccharides from Flammulina velutipes improve scopolamine-induced impairment of learning and memory of rats. J Funct Foods 18: 411-422, 2015.

(i) $(-)$ This work is licensed under a Creative Commons Attribution-NonCommercial-NoDerivatives 4.0 International (CC BY-NC-ND 4.0) License. 